\title{
Focusing on the Unfolded Protein Response and Autophagy Related Pathways to Reposition Common Approved Drugs against COVID-19
}

\author{
Serguei Nabirotchkin ${ }^{1}$, Alex E. Peluffo ${ }^{1}$, Jan Bouaziz ${ }^{1}$ and Daniel Cohen ${ }^{1, *}$ \\ 1. Pharnext, 11 rue René Jacques, 92130 Issy-les-Moulineaux, France. \\ *. Email: dcohen@pharnext.com
}

\begin{abstract}
More than 179,000 individuals have fallen ill of the Coronavirus disease (COVID-19) caused by the SARS-CoV-2 virus, which first emerged in China less than four months ago in December 2019. As of today, there exist no approved treatments against COVID-19. Vaccines are being developed, but they will take time, at least one year, to reach the population. Drug repositioning represents a fast track because already approved medicines have been broadly tested through multiple trials. We developed a repositioning strategy that mostly leads to candidates that are commonly used. The advantages are that they will facilitate proof of concept in humans through a "real-world evidence" approach and should be rapidly available to the population. We focus on the established research results that the unfolded protein response (UPR) and autophagy pathways of the host cells are essential to the life cycle of previously known coronaviruses. We performed the relevant bioinformatics analysis to understand and confirm if SARS-CoV-2 may interact with these druggable pathways. Based on these considerations, we prioritized two additional druggable pathways, which are important to the viral life cycle and tightly connected to UPR/autophagy signaling: the mitochondrial permeability transition pores (MPTP) and NLRP-3 inflammasome pathways. These four important pathways are perturbed in most major common diseases and have therefore been targeted by numerous broadly prescribed drugs. We have identified 97 approved drugs that are known to modulate these four identified pathways, and which represent, therefore, interesting repositioning candidates. Although it is indisputable that these drugs should never be used for immediate self-medication against COVID-19, we notice that some of them could also be prescribed to individuals who have COVID-19 comorbidities (e.g., hypertension). It is debated if these comorbidities are linked to the pathology itself (e.g., hypertension) or the drugs used to treat the pathology (e.g., sartans). Therefore, relevant preclinical tests and massive electronic health records (i.e., real-world evidence) must be used to pre-screen them and check the COVID-19 prognosis of individuals taking these drugs.
\end{abstract}

Keywords: COVID-2019; SARS-CoV-2; 2019-nCoV; repositioning; UPR/Autophagy; real-world evidence; pathways 


\section{Introduction}

As of March 17 th, 2020, the COVID-19 disease, caused by the SARS-CoV-2 virus (a.k.a 2019-nCoV), has become a pandemic with 179,111 confirmed cases and 7,426 deaths in more than 100 countries ${ }^{1}$. According to multiple studies, these numbers are greatly underestimated due to the lack of systematic testing and a potentially high number of asymptomatic cases ${ }^{2}$. There are no approved treatments against COVID-19, and vaccines being developed are not expected to reach patients promptly ${ }^{3}$. Repositioning of approved drugs, or which are in ongoing clinical development, is a powerful solution to rapidly yield efficient treatments for all unmet medical needs ${ }^{4,5}$. To respond to the COVID-19 emergency, multiple repositioning initiatives have been launched, most of which focus on drugs known for their antiviral activity ${ }^{6}$. Very recent work showed that the SARS-CoV virus, which triggered the 2002-2003 Severe Acute Respiratory Syndrome (SARS) epidemic, and the MERS-CoV virus, which triggered the 2012 Middle East Respiratory Syndrome (MERS) epidemic, both coronaviruses, are closely related to the SARS-CoV-2 virus ${ }^{7,8}$.

In this situation, because there doesn't exist enough functional studies of SARS-CoV-2, one possibility is to focus drug repositioning on host-cell pathways which are likely to be affected by all coronaviruses' life cycles, and which are targeted by an already existing wide range of drugs. Coronaviruses are beta-coronaviruses with a single-stranded RNA genome that encodes 16 nonstructural proteins, among which some are considered targetable such as several proteases, one helicase, and the RNA-dependent RNA-polymerase ${ }^{3}$. Beyond the protein and RNA replication machinery, classic targets of antiviral drugs ${ }^{9}$, the lifecycle of coronaviruses relies on several host-cell encoded cellular pathways.

Among those pathways, the Unfolded Protein Response (UPR) and Autophagy pathways are tightly interconnected and were shown to be essential for viral infection by multiple previous studies ${ }^{10-}$ ${ }^{13}$. Briefly, these two pathways are involved in the regulation of protein homeostasis, apoptosis, and play pivotal roles in the control of innate immunity, including the clearance of viral particles ${ }^{14,15}$. For example, recent work showed that the MERS-CoV virus induces reduced levels of the Beclin1 (BECN1) protein, which blocks the fusion of autophagosomes and lysosomes and the inhibition of the S-phase kinase-associated protein 2 (SKP2), an E3 ubiquitin ligase which promotes BECN1 degradation, enhances autophagy and reduces the replication of MERS-CoV up to 28,000-fold ${ }^{16}$. In addition, viruses are also able to modify and use the UPR/Autophagy signaling networks to complete their life cycle (e.g., enter the host cell, produce large quantities of their viral proteins) $)^{10-13}$.

Moreover, therapeutic targeting of the UPR/Autophagy pathway has attracted a lot of research interests for neurodegenerative and cancer diseases. For this reason, there exists a wide range of drugs that target these pathways ${ }^{17-19}$ and which could be immediately repositioned against COVID-19. 
In this study, we sought to assess further the putative link between COVID-19 and the UPR/autophagy pathways. To this end, we performed a bioinformatics analysis in which we relied on the aforementioned evidence that SARS-CoV-2 is closely related to the virus, which has caused the 2002-2003 SARS epidemic and to other coronaviruses. This allowed us to derive a set of host cell genes putatively involved in COVID-2019. By performing pathway enrichment analysis over those genes and identifying relevant protein-protein interactions (PPIs), we were able to list important pathways affected in infected cells. We found that several of those pathways are linked to the UPR/autophagy pathways. Furthermore, we found that a remarkably high percentage of drugs currently undergoing repositioning for COVID-19 are known modulators of the UPR/autophagy, which further supports the key role of these cellular pathways in that disease. Based on these findings, we suggest that two additional pathways, which are linked to the UPR/Autophagy pathways, are important for the viral cycle of SARS-CoV-2: the NLRP3 inflammasome signaling pathway and the pro-apoptotic mitochondrial signaling pathway. We then propose a list of drugs that are reported modulators of these four pathways, most of which are frequently prescribed and not primarily known as antiviral compounds. Finally, we indicate a global strategy to pre-screen these drugs and rapidly launch clinical trials.

\section{Materials and Methods}

\section{Cov-SARS related gene-products}

We first identified viral host gene-products (i.e., in most cases proteins) which could putatively be involved in COVID-19 by interrogating the Open Targets platform (https://www.opentargets.org/) (v3.16.1, data version 20.02) which integrates evidence from human population genetics, genomics, transcriptomics, known pathways, animal models and scientific literature data ${ }^{20}$. Importantly, the database integrates data from numerous diverse sources, and is, a priori, unbiased towards any specific pathway. Because COVID-19 is not yet a cataloged disease in this database, we searched for geneproducts associated to the following available key terms: "coronavirus infectious disease" and "severe acute respiratory syndrome" (SARS) and combined them in one single list of "Cov-SARS" geneproducts where each of them is indexed via its official symbol (Table S1).

\section{Pathway Enrichment and Protein-Protein Interaction Analyses}

We performed pathway enrichment analysis over the Cov-SARS gene-products list, a method that identifies canonical biological pathways that are significantly enriched in gene-products from this list, i.e., overrepresented, more than expected by chance ${ }^{21}$. This first pathway enrichment analysis was hypothesis-driven: based on our literature-supported assumption that UPR/autophagy plays an important role in coronaviruses infections, we searched for enriched pathways in our list of Cov-SARS 
gene-products. To this end, we used the bioinformatics tool IPA (Ingenuity Pathway Analysis v49932394 2019-11-15) (QIAGEN Inc., https://digitalinsights.qiagen.com/productsoverview/discovery-insights-portfolio/analysis-and-visualization/qiagen-ipa/ $)^{22}$ to perform a so-called "core analysis/Expression analysis" based on the Ingenuity Genes Knowledge Base reference set (Table S2). Our goal was to identify major cellular pathways putatively affected by the viral infection. We define as "statistically enriched", pathways for which the IPA reported a nominal p-value inferior to 0.05 . One difficulty with pathway enrichment analysis methods is that their results can be sensitive to the manual curation of their underlying databases (e.g., which genes are in which pathway) and statistical assumptions ${ }^{23}$.

For this reason, we performed a complementary bioinformatics inquiry based on proteinprotein interactions (PPI) referenced by IPA (Ingenuity Expert Findings + Third Party Information: BIND, Cognia, DIP, Interactome studies, MIPS, BioGRID, IntAct). Having confirmed that the UPR/autophagy linked pathways are enriched and having labelled each Cov-SARS gene to its enriched pathway (Table S2), the goal of this second analysis was to combine our Cov-SARS geneproducts and their corresponding key pathways into an abstract molecular model describing the hostcell protein network possibly impacted by the SARS-CoV-2 infection. Using our Cov-SARS geneproducts list, (1) we first identified all direct PPI among the proteins in the Cov-SARS list. When multiple proteins of the list directly interact together, we group them in what we call a "class I complex" (i.e., a component in standard graph language). (2) Secondly, we identified proteins, not contained in our list, which directly interact (via PPI) with at least three of our Cov-SARS proteins and which group together in what we call a "class II complex". (3) We retained only the class II complexes for which the non-Cov-SARS protein could be considered as a "master protein" because it belongs to an IPA canonical pathway (Ingenuity Expert Findings). (4) Finally, we connected class I complexes to the retained class II complexes by direct PPI (i.e. the class I complex and class II complex share common proteins) and/or by functional relations (i.e., metabolite, phosphorylation cascade, or any other IPA reference relation that is not a PPI), which are also documented by the IPA database, to delineate the most important and experimentally supported pathways affected by the SARS-CoV-2 infection ("Cov-SARS related pathways") (see Figure S1 and example "recognition receptor RIG1like).

\section{Drug Repositioning on candidate pathways}

We identified the candidate drugs modulating the function of our selected pathways by systematic scientific literature search (see reference in Table 2)

\section{Analysis of drugs being repositioned against COVID-19}

To evaluate the results of our pathway enrichment and PPI analyses, we retrieved the latest World Health Organization (WHO) report of drugs being clinically investigated against COVID-19 


\section{(https://www.who.int/blueprint/priority-diseases/key-}

action/Table_of_therapeutics_Appendix_17022020.pdf?ua=1) (February 17th, 2020 version) and checked if they are modulators of the UPR/Autophagy pathways (Table 1).

\section{Known antiviral status of our repositioning candidates}

Finally, for drugs identified as putatively acting on our top Cov-SARS related pathways (Table 2), we checked their therapeutic category via IPA, Drug Bank v5.1 (https://www.drugbank.ca/) and/or the Royal Pharmaceutical Society MedecinesComplete database

(https://about.medicinescomplete.com/\#/).

\section{Results and Discussion}

Using the Open Targets database, we find that 274 genes are involved in coronavirus infections (CovSARS genes, Table S1). Numerous genes on our list are directly implicated in the host cell immune response. Including, for instance, a large group of cytokines and their receptors (e.g., IL-3, IL-15, CXCL11, TNF, TNFRSF1A, CXCR3). Expectedly, we also found the ACE2 gene, which encodes a receptor, both used by the SARS-CoV and SARS-CoV-2 viruses to enter the host cell ${ }^{24}$.

Among the statistically enriched pathways $(\mathrm{p}<0.05)$, our pathway enrichment analysis identified numerous signaling pathways involved in the immune response, in particular, the following pathways: "pattern recognition receptors signaling", "interferon induction", "inflammasome pathway" (Table S2), which are directly implicated in the antiviral innate immune response ${ }^{25-28}$. This further supports the link between our Cov-SARS gene-products list and coronavirus infections. Also, among those statistically enriched pathways, we found the "endoplasmic reticulum stress pathway", the "unfolded protein response signaling," and the "phagosome maturation pathway", thereby supporting the aforementioned link between the UPR pathway and coronaviruses infections.

Then, our PPI analysis confirmed the putative role of "pattern recognition receptors signaling" and "inflammasome pathway" in coronavirus infections but also allowed us to connect these two immune pathways and UPR/autophagy signaling into a single molecular framework of the host cell viral infection (Figure S1 and S2). For instance, the NLRP3 inflammasome could be activated by the RIG-I-like and TLR4 receptors-mediated signaling but is also modulated by the autophagy flux. In turn, the autophagy pathway can be activated either by the canonical ATF4 and DDIT3(CHOP) transcription factors cascade or by the RIG-I-like receptors establishing functional feedback-loops in this signaling network ${ }^{29-36}$. Remarkably, recent publications linked the activation of NLRP3 inflammasome to cytokine storms ${ }^{37}$, which were reported to be involved in severe forms of COVID1938. The ATF4 and DDIT3(a.k.a. CHOP) transcription factors are also involved in the regulation of intrinsic (mitochondria-mediated) apoptosis pathways through the downregulation of the BCL2 
protein (which can also modulate the autophagy flux), a constitutive component of the mitochondrial permeability transition pore complex (MPTP), a core regulator of apoptosis and target for several approved drugs ${ }^{39}$. Altogether, these findings confirm the important role of the UPR/Autophagy pathways in coronavirus infections and suggest it as a target to reposition drugs against COVID-19.

Also, we find that most repositioning candidate drugs (58\%) under clinical investigation against COVID-19, reported by the World Health Organization, are also modulators of autophagy (Table 1) although most of them differ by their core mechanisms-of-action, e.g., the anti-malaria drug chloroquine, the hepatitis B/C treatment type-1-interferon 2a, and the anti-HIV drug Lopinavir, This holds for drugs that were approved for other diseases but shown to be effective against coronaviruses in experimental settings and not yet studied in clinical trials (Table S3). These findings suggest that effective antiviral drugs may not only work through their direct effect on viral proteins, such as lopinavir's action on the HIV protease, but also enhance their therapeutic effects by modulating the UPR/autophagy pathways and their related pathways.

In this context, we found a set of 97 drugs modulating the function of the four selected pathways (UPR, autophagy, the NLRP3 inflammasome, and MPTP (Table 2, Figure S1) by performing an extensive literature search (see references provided with Table 2). To our knowledge, most of them are novel repositioning candidates against COVID 19.

Importantly, this list contains both activators and inhibitors of autophagy. We hypothesize that they could be used to treat different types of COVID-19 patients. Indeed, based on our findings and the proposed framework (Figure S2), it is reasonable to hypothesize that some inhibitors of autophagy (e.g., chloroquine, currently undergoing clinical trial, cf. Table 1) would prevent the virus from entering the host cell and could thereby be used to prevent SARS-CoV-2 infections while activators of autophagy (e.g., interferon-alpha 2a/2b, also undergoing clinical trial, cf. Table 1), which would help the host cell clear the viral particles, could be used to treat severe forms of COVID-19. Remarkably, most drugs among our repositioning candidates are not known as antiviral, and most of them are known to be frequently prescribed drugs (Table 2). For instance, statins, anti-diabetics, and antagonists of the angiotensin receptor 1 , which are used to treat hyperlipidemia, type 2 diabetes, and hypertension, respectively, all of which are very common diseases. In addition, type 2 diabetes and hypertension are known to be linked to an aggravated COVID-19 prognosis ${ }^{2,40}$. Therefore, it is likely that many of the patients at risk are already taking these drugs for their primary condition, and it could facilitate real-world evidence-based analyses. Such common drugs are also highly interesting candidates because they may be immediately available in large quantities, their toxicity profile is known and eminently safe (e.g. pantoprazole, metformin, cf. Table 2), and tend to be more affordable than newly developed drugs $s^{5,41}$. 
Although it is indisputable that these drugs should never be used for immediate selfmedication against COVID-19, we notice that some of them could also be prescribed to individuals who have COVID-19 comorbidities (e.g., hypertension). It is currently unclear if these comorbidities are linked to the pathology itself (e.g., hypertension) or the drugs used to treat the pathology. For instance, drugs directly implicated in renin-angiotensin signaling (e.g., sartans) are known to increase the SARS-CoV-2 host cell receptor expression ACE2 ${ }^{42}$. It is also uncertain if this effect might aggravate COVID19 or if, by modifying the UPR/autophagy flux, these drugs might have a beneficial impact by, for instance, effectively blocking the virus entry. Therefore, relevant preclinical, experimental tests and massive electronic health records (i.e., real-world evidence ${ }^{43}$ ) must be used to pre-screen them and check whether their effect could potentially prevent, treat, or, on the contrary, aggravate COVID-19 before launching safe clinical trials. To this end, we must rapidly evaluate the COVID-19 prognosis of individuals taking these drugs, through an international effort to mutualize and provide such patient data, in order to respond to this health crisis with a potentially safe, efficient, available and affordable battery of repositioned drugs. The current COVID-19 urgency calls for an international evaluation and test of these drugs as fast as possible. 


\section{Acknowledgments}

We also thank our Pharnext colleagues, Jinchao Yu, Marina Ossokina, Zahia Touat-Hamici, Laurent Cavarec, Florian Leverve, Philippe Rinaudo and Pete Collum, for their help, as well as our colleagues and collaborators at GeneNet Pharmaceuticals Co., Ltd and at Tasly Pharmaceutical Group Co., Ltd for fruitful discussions.

\section{Competing Interests}

SN, AEP, JB and DC are employees of Pharnext S.A.

\section{Bibliography}

1. WHO. Coronavirus disease 2019 (COVID-19) Situation Report - 49. World Heal. Organ. 2019, 2633 (2020).

2. Baud, D. et al. Real estimates of mortality following COVID-19 infection. Lancet Infect. Dis. 3099, 30195 (2020).

3. Li, G. \& De Clercq, E. Therapeutic options for the 2019 novel coronavirus (2019-nCoV). Nat. Rev. Drug Discov. 19, 19-20 (2020).

4. Nabirotchkin, S. et al. Next-generation drug repurposing using human genetics and network biology. Curr. Opin. Pharmacol. 1-15 (2020). doi:10.1016/j.coph.2019.12.004

5. Pushpakom, S. et al. Drug repurposing: progress, challenges and recommendations. Nat. Rev. Drug Discov. (2018). doi:10.1038/nrd.2018.168

6. Harrison, C. Coronavirus puts drug repurposing on the fast track. Nat. Biotechnol. (2020). doi:10.1038/d41587-020-00003-1

7. Gorbalenya, A. E. et al. Severe acute respiratory syndrome-related coronavirus: The species and its viruses - a statement of the Coronavirus Study Group. 1-15 (2020). doi:10.1101/2020.02.07.937862

8. Li, X. et al. Network bioinformatics analysis provides insight into drug repurposing for COVID-2019. preprints.org (2020). doi:10.20944/preprints202003.0286.v1

9. Zumla, A., Chan, J. F. W., Azhar, E. I., Hui, D. S. C. \& Yuen, K. Y. Coronaviruses-drug discovery and therapeutic options. Nat. Rev. Drug Discov. 15, 327-347 (2016). 
10. Fung, T. S. \& Liu, D. X. Coronavirus infection, ER stress, apoptosis and innate immunity. Front. Microbiol. 5, (2014).

11. Fung, T. S. \& Liu, D. X. Human Coronavirus: Host-Pathogen Interaction. Annu. Rev. Microbiol. 73, 529-557 (2019).

12. Lim, Y., Ng, Y., Tam, J. \& Liu, D. Human Coronaviruses: A Review of Virus-Host Interactions. Diseases 4, 26 (2016).

13. Abdoli, A., Alirezaei, M., Mehrbod, P. \& Forouzanfar, F. Autophagy: The multi-purpose bridge in viral infections and host cells. Rev. Med. Virol. 28, 1-15 (2018).

14. Senft, D. \& Ronai, Z. A. UPR, autophagy, and mitochondria crosstalk underlies the ER stress response. Trends Biochem. Sci. 40, 141-148 (2015).

15. Grootjans, J., Kaser, A., Kaufman, R. J. \& Blumberg, R. S. The unfolded protein response in immunity and inflammation. Nat. Rev. Immunol. 16, 469-484 (2016).

16. Gassen, N. C. et al. SKP2 attenuates autophagy through Beclin1-ubiquitination and its inhibition reduces MERS-Coronavirus infection. Nat. Commun. 10, 2-4 (2019).

17. Kondapuram, S. K., Sarvagalla, S. \& Coumar, M. S. Targeting autophagy with small molecules for cancer therapy. J. Cancer Metastasis Treat. 2019, (2019).

18. Almanza, A. et al. Endoplasmic reticulum stress signalling - from basic mechanisms to clinical applications. FEBS J. 286, 241-278 (2019).

19. Thellung, S., Corsaro, A., Nizzari, M., Barbieri, F. \& Florio, T. Autophagy activator drugs: A new opportunity in neuroprotection from misfolded protein toxicity. Int. J. Mol. Sci. 20, (2019).

20. Carvalho-Silva, D. et al. Open Targets Platform: New developments and updates two years on. Nucleic Acids Res. 47, D1056-D1065 (2019).

21. Jassal, B. et al. The reactome pathway knowledgebase. Nucleic Acids Res. 48, D498-D503 (2020).

22. Krämer, A., Green, J., Pollard, J. \& Tugendreich, S. Causal analysis approaches in ingenuity pathway analysis. Bioinformatics 30, 523-530 (2014).

23. Tarca, A. L., Bhatti, G. \& Romero, R. A comparison of gene set analysis methods in terms of sensitivity, prioritization and specificity. PLoS One 8, (2013).

24. Zhou, P. et al. A pneumonia outbreak associated with a new coronavirus of probable bat origin. Nature 2019, (2020). 
25. Chow, K. T., Gale, M. \& Loo, Y.-M. RIG-I and Other RNA Sensors in Antiviral Immunity. Annu. Rev. Immunol. 36, 667-694 (2018).

26. Sarvestani, S. T. \& McAuley, J. L. The role of the NLRP3 inflammasome in regulation of antiviral responses to influenza A virus infection. Antiviral Res. 148, 32-42 (2017).

27. Kawai, T. \& Akira, S. Antiviral signaling through pattern recognition receptors. J. Biochem. 141, 137-145 (2007).

28. Brubaker, S. W., Bonham, K. S., Zanoni, I. \& Kagan, J. C. Innate Immune Pattern Recognition: A Cell Biological Perspective. Annual Review of Immunology 33, (2015).

29. Cai, Y. et al. Interplay of endoplasmic reticulum stress and autophagy in neurodegenerative disorders. Autophagy 12, 225-244 (2016).

30. Rashid, H. O., Yadav, R. K., Kim, H. R. \& Chae, H. J. ER stress: Autophagy induction, inhibition and selection. Autophagy 11, 1956-1977 (2015).

31. Poeck, H. et al. Recognition of RNA virus by RIG-I results in activation of CARD9 and inflammasome signaling for interleukin 1B production. Nat. Immunol. 11, 63-69 (2010).

32. Su, Q. et al. Effects of the TLR4/Myd88/NF-אB Signaling Pathway on NLRP3 Inflammasome in Coronary Microembolization-Induced Myocardial Injury. Cell. Physiol. Biochem. 47, 14971508 (2018).

33. Tsutsui, H., Imamura, M., Fujimoto, J. \& Nakanishi, K. The TLR4/TRIF-mediated activation of NLRP3 inflammasome underlies endotoxin-induced liver injury in mice. Gastroenterol. Res. Pract. 2010, (2010).

34. Houtman, J. et al. Beclin1-driven autophagy modulates the inflammatory response of microglia via NLRP 3 . EMBO J. 38, 1-15 (2019).

35. Lee, N. R. et al. Activation of RIG-I-mediated antiviral signaling triggers autophagy through the MAVS-TRAF6-Beclin-1 signaling axis. Front. Immunol. 9, 1-14 (2018).

36. Subramanian, N., Natarajan, K., Clatworthy, M. R., Wang, Z. \& Germain, R. N. The adaptor MAVS promotes NLRP3 mitochondrial localization and inflammasome activation. Cell 153, 348-361 (2013).

37. Lin, L. et al. An NLRP3 inflammasome-triggered cytokine storm contributes to streptococcal toxic shock-like syndrome (STSLS). PLoS Pathog. 15, (2019).

38. Chen, C. et al. Thalidomide Combined with Low-dose Glucocorticoid in the Treatment of COVID-19 Pneumonia. 1-6 (2020). 
39. Olszewska, A. \& Szewczyk, A. Mitochondria as a pharmacological target: Magnum overview. IUBMB Life 65, 273-281 (2013).

40. Chen, N. et al. Epidemiological and clinical characteristics of 99 cases of 2019 novel coronavirus pneumonia in Wuhan, China: a descriptive study. Lancet 395, 507-513 (2020).

41. Ashburn, T. T. \& Thor, K. B. Drug repositioning: Identifying and developing new uses for existing drugs. Nat. Rev. Drug Discov. 3, 673-683 (2004).

42. Klimas, J. et al. Perinatally administered losartan augments renal ACE2 expression but not cardiac or renal Mas receptor in spontaneously hypertensive rats. J. Cell. Mol. Med. 19, 19651974 (2015).

43. Sherman, R. E. et al. Real-world evidence - What is it and what can it tell us? N. Engl. J. Med. 375, 2293-2297 (2016).

\section{Tables}

Table 1. Drugs ongoing COVID-19 preclinical and clinical development according to the World Health Organization, whether they modulate the UPR/Autophagy pathways and the supporting published evidence (listed below by corresponding reference number). For Thalidomide, although the drug does not induce autophagy itself, it enhances the pro-autophagic effect of another drug.

\begin{tabular}{|l|c|c|}
\hline \multicolumn{1}{|c|}{ Drug name } & UPR/autophagy modulator & Reference(s) \\
\hline Interferon alfa-2a / 2b & yes & 1 \\
\hline Chloroquine & yes & 2 \\
\hline Corticosteroids & yes & 3 \\
\hline Darunavir & no & - \\
\hline Cobicistat & no & - \\
\hline Emtricitabine & yes & 5 \\
\hline Tenofovir & yes & 7 \\
\hline Ruxolitinib & yes & 8 \\
\hline Rapamycin & yes & 9 \\
\hline Mycophenolate mofetil & yes & 1 \\
\hline FN-B1a (IFNB1) & yes & 10,11 \\
\hline IFN-gamma & yes & 12 \\
\hline Ribavirin & yes & 13,14 \\
\hline Lopinavir & yes & 15 \\
\hline Ritonavir & yes & - \\
\hline Baloxavir marboxil & no data & - \\
\hline Favipiravir & no data & - \\
\hline Enisamium iodide & no data & - \\
\hline Arbidol (Umifenovir) & no data & \\
\hline
\end{tabular}




\begin{tabular}{|c|c|c|}
\hline Remdesivir & no data & - \\
\hline IFN- $\lambda 1 \mathrm{a}$ & yes & 16,17 \\
\hline Thalidomide & yes * & 18 \\
\hline Camostat & yes & 19 \\
\hline $\mathrm{BCX} 4430$ & no data & - \\
\hline Relacatib & no data & - \\
\hline Lycorine & yes & 20 \\
\hline Asterivir & no data & - \\
\hline UDA/lectin & no data & - \\
\hline Hiltonol Poly-IC:LC (poly IC) & yes & 21 \\
\hline RTD-1 peptide & no data & - \\
\hline $\begin{array}{l}\mathrm{NHC}(\text { EIDD-1931) - } \beta-\mathrm{D}-\mathrm{N} 4 \text { - } \\
\text { hydroxycytidine }\end{array}$ & no data & - \\
\hline
\end{tabular}

\section{Supporting References for Table 1.}

1. Tian $Y$ et al. Crosstalk between Autophagy and Type I Interferon Responses in Innate Antiviral Immunity. Viruses. 2019 Feb 1;11(2).

2. Panda PK et al. Chemical Screening Approaches Enabling Drug Discovery of Autophagy Modulators for Biomedical Applications in Human Diseases. Front Cell Dev Biol. 2019 Mar 19; 7:38.

3. Wang J et al. Glucocorticoids Suppress Antimicrobial Autophagy and Nitric Oxide Production and Facilitate Mycobacterial Survival in Macrophages. Sci Rep. 2017 Apr 20;7(1):982.

4. Anna-Mart Engelbrecht A-M. \& Loos B. Glucocorticoid-Induced Cardioprotection: A Novel Role for Autophagy. DOI: 10.5772/52406

5. Cheney L. et al. Aberrant Autophagy in Macrophages and Astrocytes after HIV Nef or Antiretroviral Treatment: Contribution to the Pathogenesis of HIV-associated Neurocognitive Disorders. Open Forum Infect Dis. 2017 Fall; 4(Suppl 1): S208.

6. Mellor C. Developing safer HIV drugs: Elucidating the mechanistic pathway of Tenofovir-induced cytotoxicity via mitochondrial dysfunction (http://livrepository.liverpool.ac.uk/id/eprint/17093)

7. Ishida S et al. Mechanisms for mTORC1 activation and synergistic induction of apoptosis by ruxolitinib and BH3 mimetics or autophagy inhibitors in JAK2-V617F-expressing leukemic cells including newly established PVTL-2. Oncotarget. 2018 Jun 1;9(42):26834-26851.

8. Thellung S et al. Autophagy Activator Drugs: A New Opportunity in Neuroprotection from Misfolded Protein Toxicity. Int J Mol Sci. 2019 Feb 19;20(4).

9. Fang $\mathrm{S}$ et al. Suppression of autophagy by mycophenolic acid contributes to inhibition of HCV replication in human hepatoma cells. Sci Rep. 2017 Mar 9; 7:44039.

10. Li P et al. Interferon- $y$ induces autophagy with growth inhibition and cell death in human hepatocellular carcinoma (HCC) cells through interferon-regulatory factor-1 (IRF-1). Cancer Lett. 2012 Jan 28;314(2):213-22.

11. Matsuzawa $T$ et al. Autophagy activation by interferon- $y$ via the $p 38$ mitogen-activated protein kinase signalling pathway is involved in macrophage bactericidal activity. Immunology. 2014 Jan;141(1):61-9.

12. Isakovic AM et al. Autophagy suppression sensitizes glioma cells to IMP dehydrogenase inhibition-induced apoptotic death. Exp Cell Res. 2017 Jan 1;350(1):32-40.

13. Zha BS et al. HIV protease inhibitors disrupt lipid metabolism by activating endoplasmic reticulum stress and inhibiting autophagy activity in adipocytes. PLoS One. 2013;8(3): e59514.

14. Djedaini $M$ et al. Lopinavir co-induces insulin resistance and ER stress in human adipocytes. Biochem Biophys Res Commun. 2009 Aug 14;386(1):96-100.

15. Zhou $\mathrm{H}$ et al. HIV protease inhibitors activate the unfolded protein response in macrophages: implication for atherosclerosis and cardiovascular disease. Mol Pharmacol. 2005 Sep;68(3):690-700.

16. Gao D et al. Role of autophagy in inhibiting the proliferation of A549 cells by type III interferon. Cell Biol Int. 2019 Jun;43(6):605-612.

17. Gao $D$ et al. Interferon- $\lambda 1$ suppresses invasion and enhances autophagy in human osteosarcoma cell. Int J Clin Exp Med. 2015 Sep 15;8(9):14999-5004.

18. Gao $\mathrm{S}$ et al. Mechanism of thalidomide to enhance cytotoxicity of temozolomide in U251-MG glioma cells in vitro. Chin Med J (Engl). 2009 Jun 5;122(11):1260-6.

19. Crozier SJ et al. CCK-induced pancreatic growth is not limited by mitogenic capacity in mice. Am J Physiol Gastrointest Liver Physiol. 2008 May;294(5): G1148-57. 
20. Roy M et al. Lycorine Downregulates HMGB1 to Inhibit Autophagy and Enhances Bortezomib Activity in Multiple Myeloma. Theranostics. 2016 Sep 24;6(12):2209-2224.

21. Tormo $D$ et al. Targeted activation of innate immunity for therapeutic induction of autophagy and apoptosis in melanoma cells. Cancer Cell. 2009 Aug 4;16(2):103-14. 
Table 2. Full list of the repositioning candidates $(n=97)$, and for each drug: the targeted Cov-SARS related pathway, its mechanism of action, its therapeutic category, and the supporting published evidence (listed below by corresponding reference number). In some instances, we kept on our list drugs targeting the same targets (e.g., Temsirolimus, Tacrolimus, Everolimus) because they could have different side-effects profiles. In addition, and especially for further experimental investigations, mechanisms of action mentioned for each drug should be considered with caution since the functional effects of a drug can be influenced by experimental settings, especially the cell type selected for drug screening.

\begin{tabular}{|c|c|c|c|}
\hline CANDIDATE DRUG & $\begin{array}{l}\text { MECHANISM OF } \\
\text { ACTION }\end{array}$ & THERAPEUTIC CATEGORY & REFERENCE(S) \\
\hline Rapamycin & activator of autophagy & immunosuppressant; anticancer agent & 1 \\
\hline Temsirolimus & activator of autophagy & anticancer agent & 1 \\
\hline Tacrolimus & activator of autophagy & immunosuppressant & 1 \\
\hline Everolimus & activator of autophagy & immunosuppressant; anticancer agent & 1 \\
\hline Metformin & activator of autophagy & antidiabetic drug & 1 \\
\hline Trehalose & activator of autophagy & dietary supplement & 1 \\
\hline Resveratrol & $\begin{array}{l}\text { activator of autophagy } \\
\text { and mitophagy }\end{array}$ & dietary supplement & 1 \\
\hline Lithium & activator of autophagy & antipsychotic agent; mood stabilizers & 1 \\
\hline Carbamazepine & activator of autophagy & antiepileptic drug; analgesic agent & 1 \\
\hline Sodium valproate & activator of autophagy & antiepileptic drug; mood stabilizers & 1 \\
\hline Verapamil & activator of autophagy & $\begin{array}{l}\text { antianginal drug; antiarrhythmic; } \\
\text { antihypertensive }\end{array}$ & 1 \\
\hline Nimodipine & activator of autophagy & cardiovascular drug & 1 \\
\hline Nitrendipine & activator of autophagy & antihypertensive drug & 1 \\
\hline Fluspirilene & activator of autophagy & antipsychotic drug & 2 \\
\hline Trifluoperazine & activator of autophagy & antipsychotic drug & 2 \\
\hline Nicardipine & activator of autophagy & antianginal drug; antihypertensive drug & 2 \\
\hline Tamoxifen & activator of autophagy & hormonal antineoplastic drug & 2 \\
\hline Pimozide & activator of autophagy & antipsychotic drug & 2 \\
\hline Amiodarone & activator of autophagy & antianginal drug; antiarrhythmic drug & 2 \\
\hline Loperamide & activator of autophagy & antidiarrheal drug & 2 \\
\hline Rilmenidine & activator of autophagy & antihypertensive drug & 3 \\
\hline Clonidine & activator of autophagy & $\begin{array}{l}\text { antihypertensive drug; non-narcotic } \\
\text { analgesic }\end{array}$ & 3 \\
\hline Calcifediol & activator of autophagy & calcium regulator; vitamin source drug & 3 \\
\hline Nitazoxanide & activator of autophagy & anti-infective agent & 3 \\
\hline Nortriptyline & activator of autophagy & tricyclic antidepressant & 3 \\
\hline Simvastatin & activator of autophagy & anticholesteremic agent & 3 \\
\hline Minoxidil & activator of autophagy & $\begin{array}{l}\text { antihypertensive drug; vasodilator; } \\
\text { antialopecia drug }\end{array}$ & 4 \\
\hline Bromperidol & activator of autophagy & antipsychotic drug & 4,5 \\
\hline Metergoline & activator of autophagy & CNS drug; anti-hyperprolactinemic agent & 4,5 \\
\hline Chlorpromazine & activator of autophagy & antiemetic drug; antipsychotic drug & 4,5 \\
\hline
\end{tabular}




\begin{tabular}{|c|c|c|c|}
\hline Fludrocortisone & activator of autophagy & mineralocorticoid replacement agent & 4,5 \\
\hline Noscapine & activator of autophagy & antitussive drug & 4,5 \\
\hline Clemastine & activator of autophagy & anti-allergic agent; antipruritics & 4 \\
\hline Deferiprone & activator of autophagy & chelating agent; metabolic disorder drug & 6 \\
\hline Temozolomide & activator of autophagy & alkylating antineoplastic drug & 7 \\
\hline Gefitinib & activator of autophagy & anticancer drug & 7 \\
\hline Sertindole & activator of autophagy & antipsychotic drug & 8 \\
\hline Olanzapine & activator of autophagy & antipsychotic drug & 8 \\
\hline Fluphenazine & activator of autophagy & antipsychotic drug & 8 \\
\hline Methotrimeprazine & activator of autophagy & antipsychotic drug & 8 \\
\hline Niclosamide & activator of autophagy & antihelmintic drug & 9 \\
\hline Prochlorperazine & activator of autophagy & antiemetic drug; antipsychotic drug & 5 \\
\hline Clozapine & modulator of autophagy & antipsychotic drug & 8 \\
\hline Baclofen & modulator of autophagy & skelatal muscle relaxant & 10 \\
\hline Quinacrine & inhibitor of autophagy & antihelmintic drug; antiprotozoal drug & 11,12 \\
\hline Bortezomib & inhibitor of autophagy & anticancer agent & 7 \\
\hline Pantoprazole & inhibitor of autophagy & gastrointestinal drug & 7 \\
\hline Celecoxib & inhibitor of autophagy & $\begin{array}{l}\text { non-narcotic analgesic; anticancer agent; } \\
\text { NSAID }\end{array}$ & 7 \\
\hline Azithromycin & inhibitor of autophagy & macrolide antibiotic & 4 \\
\hline Verteporfin & inhibitor of autophagy & ocular drug & 4 \\
\hline Clomipramine & inhibitor of autophagy & tricyclic antidepressant & 4 \\
\hline Chloroquine & inhibitor of autophagy & antiprotozoal drug & 3 \\
\hline Hydroxychloroquine & inhibitor of autophagy & antiprotozoal drug & 3 \\
\hline CANDIDATE DRUG & $\begin{array}{c}\text { MECHANISM OF } \\
\text { ACTION }\end{array}$ & THERAPEUTIC CATEGORY & REFERENCES \\
\hline Phenylbutyrate & suppressor of UPR stress & urea cycle disorder agent & 7,13 \\
\hline Pioglitazone & suppressor of UPR stress & antidiabetic drug & 13 \\
\hline Isoproterenol & suppressor of UPR stress & bronchodilator; cardiotonic agent & 13 \\
\hline Pravastatin & suppressor of UPR stress & hypolipidemic drug & 13 \\
\hline Etanercept & suppressor of UPR stress & antiarthritic drug; immunomodulator & 13 \\
\hline Sunitinib & suppressor of UPR stress & anticancer agent & 13 \\
\hline Curcumin & suppressor of UPR stress & dietary supplement & 13 \\
\hline Exenatide & suppressor of UPR stress & antidiabetic drug & 14 \\
\hline Vildagliptin & suppressor of UPR stress & antidiabetic drug & 14 \\
\hline Fenofibrate & suppressor of UPR stress & hypolipidemic drug & 14 \\
\hline Valsartan & suppressor of UPR stress & antihypertensive drug & 14 \\
\hline Losartan & suppressor of UPR stress & antihypertensive drug & 14 \\
\hline Olmesartan & suppressor of UPR stress & antihypertensive drug & 14 \\
\hline Telmisartan & suppressor of UPR stress & $\begin{array}{l}\text { antihypertensive drug; cardiovascular } \\
\text { agent }\end{array}$ & 14 \\
\hline Guanabenz & suppressor of UPR stress & antihypertensive drug & 15 \\
\hline Trazodone & suppressor of UPR stress & antidepressant & 16 \\
\hline Berberine & suppressor of UPR stress & antimicrobial agent; antidiabetic agent & 17 \\
\hline Bisoprolol & suppressor of UPR stress & $\begin{array}{l}\text { antihypertensive drug; cardiovascular } \\
\text { agent }\end{array}$ & 17 \\
\hline Propranolol & suppressor of UPR stress & $\begin{array}{l}\text { antihypertensive drug; antianginal drug; } \\
\text { antiarrhythmic drug }\end{array}$ & 17 \\
\hline Metoprolol & suppressor of UPR stress & antianginal drug; antihypertensive drug & 17 \\
\hline
\end{tabular}




\begin{tabular}{|c|c|c|c|}
\hline Atorvastatin & UPR stress modulator & anticholesteremic agent & 17 \\
\hline Liraglutide & UPR stress modulator & antidiabetic drug; anti-obesity agent & 17 \\
\hline Alpha-lipoic acid & UPR stress modulator & $\begin{array}{l}\text { nutritional supplement; antioxidants; anti- } \\
\text { neuropathic agent }\end{array}$ & 17 \\
\hline Melatonin & UPR stress modulator & CNS drug; anti-insomnia agent & 18 \\
\hline Quercetin & UPR stress modulator & dietary supplement & 19 \\
\hline CANDIDATE DRUG & $\begin{array}{c}\text { MECHANISM OF } \\
\text { ACTION }\end{array}$ & THERAPEUTIC CATEGORY & REFERENCES \\
\hline Haloperidol & MPTP modulator & antipsychotic drug; antiemetic drug & $20,21,22$ \\
\hline Pentazocine & MPTP modulator & narcotic analgesic & $20,21,22$ \\
\hline Ifenprodil & MPTP modulator & vasodilator & $20,21,22$ \\
\hline Donepezil & MPTP modulator & anti-Alzheimer's drug; CNS drug & $20,21,22$ \\
\hline Carbetapentane & MPTP modulator & antitussive & $20,21,22$ \\
\hline Dextromethorphan & MPTP modulator & antitussive & $20,21,22$ \\
\hline Edaravone & MPTP modulator & neuroprotective agent; anti-ALS drug & 23,24 \\
\hline Cyclosporin A & MPTP modulator & immunosuppressant & $24,25,26$ \\
\hline Diazoxide & MPTP modulator & antihypertensive drug; vasodilator & $24,25,26$ \\
\hline Nicorandil & MPTP modulator & antianginal drug & $24,25,26$ \\
\hline Tadalafil & MPTP modulator & impotence drug; antihypertensive drug & $24,25,26$ \\
\hline Perhexiline & MPTP modulator & antianginal drug & $24,25,26$ \\
\hline Carvedilol & MPTP modulator & antihypertensive drug & $24,25,26$ \\
\hline Etifoxine & MPTP modulator & antipsychotic drug & 27,28 \\
\hline Pramipexole & MPTP modulator & antiparkinsonian drug & 29 \\
\hline CANDIDATE DRUG & $\begin{array}{c}\text { MECHANISM OF } \\
\text { ACTION }\end{array}$ & THERAPEUTIC CATEGORY & REFERENCES \\
\hline Glyburide & $\begin{array}{l}\text { NLRP3 Inflammasome } \\
\text { inhibitor }\end{array}$ & antidiabetic drug & 30,31 \\
\hline Tranilast & $\begin{array}{l}\text { NLRP3 Inflammasome } \\
\text { inhibitor }\end{array}$ & anti-inflammatory agent; & 31,32 \\
\hline Anakinra & $\begin{array}{l}\text { NLRP3 Inflammasome } \\
\text { inhibitor }\end{array}$ & antiarthritic drug; antiinflammatory agent & 31 \\
\hline Thalidomide & $\begin{array}{l}\text { NLRP3 Inflammasome } \\
\text { inhibitor }\end{array}$ & $\begin{array}{l}\text { anticancer agent; antiemetic drug; } \\
\text { immunomodulator }\end{array}$ & 31 \\
\hline
\end{tabular}

\section{Supporting References for Table 2.}

1. Thellung S et al. Autophagy Activator Drugs: A New Opportunity in Neuroprotection from Misfolded Protein Toxicity. Int J Mol Sci. 2019 Feb 19;20(4).

2. Zhang $L$ et al. Small molecule regulators of autophagy identified by an image-based high-throughput screen. Proc Natl Acad Sci U S A. 2007 Nov 27;104(48):19023-8.

3. Panda PK et al. Chemical Screening Approaches Enabling Drug Discovery of Autophagy Modulators for Biomedical Applications in Human Diseases. Front Cell Dev Biol. 2019 Mar 19; 7:38.

4. Vakifahmetoglu-Norberg $\mathrm{H}$ et al. Pharmacologic agents targeting autophagy. J Clin Invest. 2015 Jan;125(1):5-13.

5. Shaw SY et al. Selective modulation of autophagy, innate immunity, and adaptive immunity by small molecules. ACS Chem Biol. 2013 Dec 20;8(12):2724-2733.

6. Allen GF et al. Loss of iron triggers PINK1/Parkin-independent mitophagy. EMBO Rep. 2013 Dec;14(12):1127-35.

7. Kondapuram SK et al. Targeting autophagy with small molecules for cancer therapy. Cancer Metastasis Treat 2019; 5:32.

8. Vucicevic $L$ et al. Mechanisms and therapeutic significance of autophagy modulation by antipsychotic drugs. Cell Stress. 2018 Oct 25;2(11):282-291.

9. Renna $\mathrm{M}$ et al. Chemical inducers of autophagy that enhance the clearance of mutant proteins in neurodegenerative diseases. J Biol Chem. 2010 Apr 9;285(15):11061-7. 
10. Liu L Et al. Baclofen mediates neuroprotection on hippocampal CA1 pyramidal cells through the regulation of autophagy under chronic cerebral hypoperfusion. Sci Rep. 2015 Sep 28; 5:14474.

11. Goodall ML et al. Development of potent autophagy inhibitors that sensitize oncogenic BRAF V600E mutant melanoma tumor cells to vemurafenib. Autophagy. 2014 Jun;10(6):1120-36.

12. Khurana A et al. Quinacrine promotes autophagic cell death and chemosensitivity in ovarian cancer and attenuates tumor growth. Oncotarget. 2015 Nov 3;6(34):36354-69.

13. Minamino $\mathrm{T}$ et al. Endoplasmic reticulum stress as a therapeutic target in cardiovascular disease. Circ Res. 2010 Oct 29;107(9):1071-82.

14. Jung TW \& Choi KM. Pharmacological Modulators of Endoplasmic Reticulum Stress in Metabolic Diseases. Int J Mol Sci. 2016 Feb 1;17(2).

15. Tsaytler $\mathrm{P}$ et al. Selective inhibition of a regulatory subunit of protein phosphatase 1 restores proteostasis. Science. 2011 Apr 1;332(6025):91-4.

16. Pérez-Arancibia $R$ et al. (off)Targeting UPR signaling: the race toward intervening ER proteostasis. Expert Opin Ther Targets. 2018 Feb;22(2):97-100.

17. Amen $\mathrm{OM}$ et al. Endoplasmic Reticulum Stress Activates Unfolded Protein Response Signaling and Mediates Inflammation, Obesity, and Cardiac Dysfunction: Therapeutic and Molecular Approach. Front Pharmacol. 2019 Sep 10; 10:977.

18. Almanza A et al. Endoplasmic reticulum stress signalling - from basic mechanisms to clinical applications. FEBS J. 2019 Jan;286(2):241-278.

19. Rivas A et al. Targeting the unfolded protein response for disease intervention. Expert Opin Ther Targets. 2015;19(9):1203-18.

20. Christ MG et al. The Sigma-1 Receptor at the Crossroad of Proteostasis, Neurodegeneration, and Autophagy. Trends Neurosci. 2020 Feb ;43(2):79-81.

21. Hayashi $T$ et al. Targeting ligand-operated chaperone sigma-1 receptors in the treatment of neuropsychiatric disorders. Expert Opin Ther Targets. 2011 May;15(5):557-77.

22. Albayrak Y \& Hashimoto K. Sigma-1 Receptor Agonists and Their Clinical Implications in Neuropsychiatric Disorders. Adv Exp Med Biol. 2017; 964:153-161.

23. Takayasu $\mathrm{Y}$ et al. Edaravone, a radical scavenger, inhibits mitochondrial permeability transition pore in rat brain. J Pharmacol Sci. 2007 Apr;103(4):434-7.

24. Olszewska A \& Szewczyk A. Mitochondria as a pharmacological target: magnum overview. IUBMB Life. 2013 Mar;65(3):273-81.

25. Javadov $S$ \& Karmazyn M. Mitochondrial permeability transition pore opening as an endpoint to initiate cell death and as a putative target for cardioprotection. Cell Physiol Biochem. 2007;20(1-4):1-22.

26. Pourahmad J et al. Mitochondrial Targeting for Drug Development, DOI: 10.5772/59719.

27. Liere $P$ et al. Differential effects of the $18-\mathrm{kDa}$ translocator protein (TSPO) ligand etifoxine on steroidogenesis in rat brain, plasma and steroidogenic glands: Pharmacodynamic studies. Psychoneuroendocrinology. 2017 Sep ;83:122-134.

28. Costa B et al. The Anxiolytic Etifoxine Binds to TSPO Ro5-4864 Binding Site with Long Residence Time Showing a High Neurosteroidogenic Activity. ACS Chem Neurosci. 2017 Jul 19;8(7):1448-1454.

29. Ferrari-Toninelli $G$ et al. Mitochondria-targeted antioxidant effects of $S(-)$ and $R(+)$ pramipexole. BMC Pharmacol. 2010 Feb 5; 10:2.

30. Zahid A et al. Pharmacological Inhibitors of the NLRP3 Inflammasome. Front Immunol. 2019 Oct 25; 10:2538.

31. Xu S et al. Inflammasome inhibitors: promising therapeutic approaches against cancer. J Hematol Oncol. 2019 Jun 26;12(1):64.

32. Huang $\mathrm{Y}$ et al. Tranilast directly targets NLRP3 to treat inflammasome-driven diseases. EMBO Mol Med. 2018 Apr;10(4). 


\section{List of Supplementary Figures and Tables}

Table S1. List of Open Targets derived Cov-SARS gene-products referenced via their official symbol and ordered alphabetically.

Table S2. IPA pathway enrichment analysis (core analysis/expression analysis). We give the IPA canonical pathway names, the p-value of a Fisher's exact test (which test the enrichment of the pathway in our Cov-SARS gene-products list compared to the null hypothesis of the enrichment of the same pathway in all the genes cataloged by IPA) and the Cov-SARS gene-products which belong to that pathway. Finally, we highlight in yellow pathways related to the UPR/Autophagy pathways.

Table S3. Drug effective against other coronaviruses (SARS-CoV, MERS-CoV), not in clinical development, whether they modulate the UPR/Autophagy pathways and the supporting published evidence.

Figure S1. Summary of the bioinformatics search approach, using the RIG-I-Like receptor signaling pathway as an example.

Figure S2. The four Cov-SARS related pathways putatively involved in the viral infection. The corresponding Cov-SARS proteins are in blue, the non-Cov-SARS master proteins from the IPA canonical pathways are in grey. Between proteins, up-regulation interactions are shown with blue arrows. Down-regulations are shown in red, and modulations (i.e., up or down) are shown in black. The RIG-I-Like receptor signaling module from Figure S1 is shown next to its corresponding pathway. 